\title{
A Multilevel Subtree Method for Single and Batched Sparse Cholesky Factorization
}

\author{
Meng Tang \\ CISE, University of Florida \\ USA \\ mtang@cise.ufl.edu
}

\author{
Mohamed Gadou \\ CISE, University of Florida \\ USA \\ mgadou@cise.ufl.edu
}

\author{
Steven C. Rennich \\ NVIDIA \\ Santa Clara, CA, USA \\ srennich@nvidia.com
}

\author{
Timothy A. Davis \\ CSE, Texas A\&M University \\ USA \\ davis@tamu.edu
}

\author{
Sanjay Ranka \\ CISE, University of Florida \\ USA \\ ranka@cise.ufl.edu
}

\begin{abstract}
Scientific computing relies heavily on matrix factorization. Cholesky factorization is typically used to solve the linear equation system $A x=b$ where $A$ is symmetric and positive definite. A large number of applications require operating on sparse matrices. A major overhead with factorization of sparse matrices on GPUs is addressing the cost of transferring the data from the CPU to the GPU. Additionally, the computational efficiency of factorization of small dense matrices has to be addressed.

In this paper, we develop a multilevel subtree method for Cholesky factorization of large sparse matrices on single and multiple GPUs. This approach effectively addresses two important limitations of previous methods. First, by applying the subtree method to both lower levels and higher levels of the elimination tree, we improve the amount of concurrency and the computational efficiency. Previous approaches only used the subtree method at the lower levels. Second, we overlap computation of a subtree with another subtree, thereby reducing the overhead of the data transfer from CPU to GPU. Additionally, we propose the use of batched parallelism for applications that require simultaneous factorization of multiple matrices. Effectively, the tree structure of a collection of matrices can be derived by merging the individual trees.

Our experimental results show that each of the three techniques result in significant performance improvement. Further, the combination of the three can result in a speedup of up to 2.43 on a variety of sparse matrices.
\end{abstract}

\section{CCS CONCEPTS}

- Computing methodologies $\rightarrow$ Factorization methods;

\footnotetext{
Permission to make digital or hard copies of all or part of this work for personal or classroom use is granted without fee provided that copies are not made or distributed for profit or commercial advantage and that copies bear this notice and the full citation on the first page. Copyrights for components of this work owned by others than the author(s) must be honored. Abstracting with credit is permitted. To copy otherwise, or republish, to post on servers or to redistribute to lists, requires prior specific permission and/or a fee. Request permissions from permissions@acm.org.

ICPP 2018, August 13-16, 2018, Eugene, OR, USA

(c) 2018 Copyright held by the owner/author(s). Publication rights licensed to ACM. ACM ISBN 978-1-4503-6510-9/18/08 . .\$15.00

https://doi.org/10.1145/3225058.3225090
}

\section{KEYWORDS}

sparse matrices, sparse direct methods, Cholesky factorization, GPU, CUDA

ACM Reference Format:

Meng Tang, Mohamed Gadou, Steven C. Rennich, Timothy A. Davis, and Sanjay Ranka. 2018. A Multilevel Subtree Method for Single and Batched Sparse Cholesky Factorization. In ICPP 2018: 47th International Conference on Parallel Processing, August 13-16, 2018, Eugene, OR, USA. ACM, New York, NY, USA, 10 pages. https://doi.org/10.1145/3225058.3225090

\section{INTRODUCTION}

Sparse direct methods are used to solve linear algebraic problems [3] including systems of linear equations, sparse linear squares and eigenvalue problems, and form the backbone of many applications in computational science [18]. The computational workflow of sparse Cholesky factorization [4] is structured as a tree, also known as the elimination tree. Each node in the elimination tree is called a supernode. A supernode represents factorization of a dense sub-matrix, whereas an edge represents an update operation of a supernode by one of its factorized descendants. A supernode, before being factorized, must be updated by all its relevant descendants. Further, sub-matrices with no children or those that have been already updated by all relevant descendants may be factorized in parallel.

Hybrid multicore processors (HMPs) are poised to dominate the landscape of the next generation of computing on the desktop as well as on exascale systems. HMPs consist of general purpose CPU and GPU cores along with specialized cores and are expected to provide benefits to a wide spectrum of applications at significantly lower energy requirements per floating-point operation.

In this paper, we describe efficient multithreaded and pipelined algorithms for sparse Cholesky factorization on HMPs consisting of CPUs and GPUs. This algorithm is shown to provide significant improvement over the baseline supernodal algorithm, CHOLMOD, in both its CPU [2] and GPU [14] versions. CHOLMOD is a SuiteSparse module provided by Chen, Davis, Hager and Rajamanickam, capable of performing sparse Cholesky factorization and widely used. The first GPU based CHOLMOD Version 4.0.0 [13] was improved in a later version 4.5.5, using a baseline GPU algorithm, and further extended using the "subtree" algorithm, in version 4.6.0-beta. We will refer to the 4.6.0-beta version as the "single level" subtree 
algorithm in this paper. The baseline, as well as the subtree algorithm, is left-looking supernodal. The authors in [16] improved the performance of baseline CHOLMOD using multiple CPU threads and pipelining.

In this paper, we develop a multilevel subtree method for Cholesky factorization of large sparse matrices on single and multiple GPUs. This approach effectively addresses two important limitations of previous methods. First, by applying the subtree method to both lower levels and higher levels of the elimination tree, we improve the amount of concurrency and the computational efficiency. We also reduce the number of kernel launches (and the corresponding overhead). Previous approaches only used the subtree method at the lower levels. Second, we overlap computation of a subtree with another subtree, thereby reducing the overhead of the data transfer from CPU to GPU. Additionally, we propose the use of batched parallelism for applications that require simultaneous factorization of multiple matrices. Effectively, the tree structure of a collection of matrices can be derived by merging the individual trees. This improves efficiency on single and multiple GPUs.

For our experiments, we used sparse matrices from the SuiteSparse Matrix Collection [5]. The results demonstrate up to $142 \%$ improvement in performance with the application of multilevel subtree algorithm, and an additional $140 \%$ improvement in performance with the application of batched sparse Cholesky factorization compared to one-by-one matrix factorization over multiple GPUs.

Section 2 briefly introduces related research and techniques for sparse Cholesky factorization. The left-looking supernodal Cholesky algorithm and the workflow of CHOLMOD are described in detail in Section 3. The subtree algorithm is described in Section 4 whereas the multilevel subtree algorithm is described in in Section 5. Section 6 gives an introduction to our implementation of the batched sparse Cholesky algorithm. Experimental results are presented in Section 7. Finally, we conclude in Section 8.

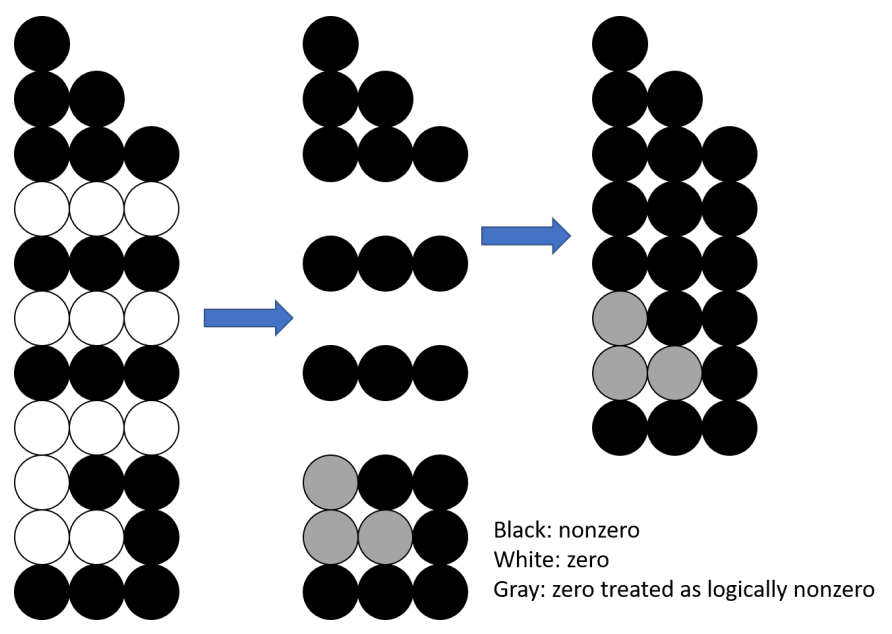

Figure 1: Construction of a supernode of the elimination tree in the supernodal Cholesky algorithm

\section{RELATED WORK}

Large-scale sparse matrix factorization is an important topic and many scientific computing applications such as Google Street View

and finite element methods rely on it. Multicore architectures have been an important tool being used to carry out the intensive computations required in matrix factorization.

Cholesky factorization is one of the factorization techniques being used. Supernodal Cholesky factorization was first introduced by Ashcraft et al. [1]; see also Section 9.1 of [6] for a survey of the method. Lacoste et al. [12] describe a GPU-accelerated supernodal Cholesky factorization where supernodes are assembled on the CPU, and Hogg et al. [10] present a GPU-accelerated multifrontal factorization where frontal matrices are assembled on the GPU instead.

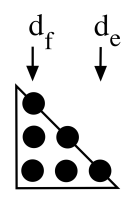

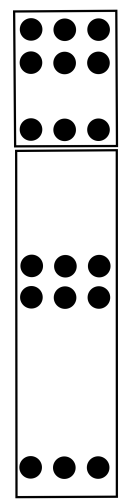

d: descendant supernode

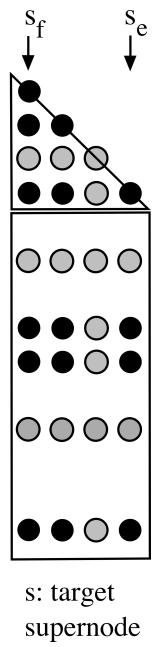

Figure 2: Updating a supernode by its relevant descendants in supernodal Cholesky algorithm

Chen et al. [2] proposed a supernodal algorithm for sparse Cholesky factorization, CHOLMOD, on a multicore system equipped with multiple CPUs and another implementation of CHOLMOD was provided on HMPs with GPU [14]. Tang et al. extended this work and provided an optimized version of CHOLMOD on GPU [15]. The work provided in $[14,15]$ was on a single GPU and when we tried to extend it to multiple GPUs to carry out the factorization, the computations were not enough to utilize all the GPUs and even for large sparse matrices, a single GPU was able to perform the factorization efficiently. Hence to utilize a system with multiple GPUs, batch factorization would be helpful considering that many of the scientific applications we mentioned actually do multiple sparse matrix factorization.

Researchers have previously proposed batched computations on the GPU but their work are mainly for dense matrix operations. Villa et al. [17] proposed techniques for batch LU factorization of small dense matrices of size up to $128 \times 128$ on the GPU. Kurzak et al. [11] provided an implementation for batch dense Cholesky factorization on NVIDIA GPUs where matrices are of the same sizes and 
range in dimensions from $5 \times 5$ to $100 \times 100$. Dong et al. [7] provided three different algorithms for batch Cholesky factorization on a GPU and they were able to get a speedup of $1.8 \mathrm{x}$ compared to MKL library implementation. Haidar et al. [9] provided a technique for batch QR factorization of small dense matrices on GPUs. They were able to get a $5 \mathrm{x}$ speedup compared to a batched $\mathrm{QR}$ factorization functionality provided by CUBLAS library. Haidar et al. [8] also proposed different optimization techniques for batched BLAS kernels that can be used in different factorizations namely LU, QR, and Cholesky. The above work on batch matrix factorization on GPUs is limited to dense matrices. In our work, we extend these techniques for sparse matrix factorization.

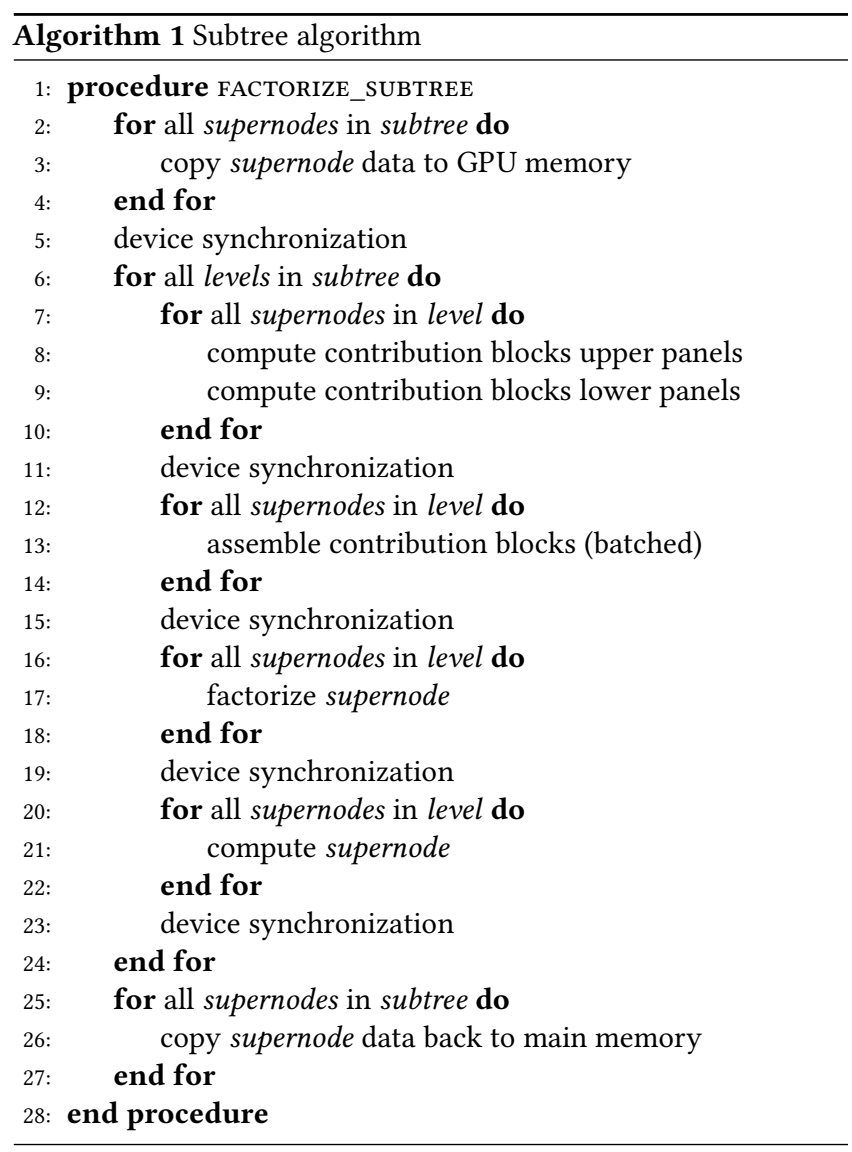

\section{SUPERNODAL SPARSE CHOLESKY FACTORIZATION}

Our work is based on the CHOLMOD package. CHOLMOD is an implementation of both the up-looking Cholesky algorithm and the left-looking supernodal Cholesky algorithm for sparse symmetric positive-definite matrices. The attributes (size, sparsity, etc.) of the matrix being factorized will determine which algorithm should be used. Our work is an optimization for CHOLMOD's left-looking supernodal Cholesky algorithm.

The workflow of a sparse Cholesky factorization is structured as an elimination tree, where each edge stands for the parent's data dependency on the children. A node in the elimination tree

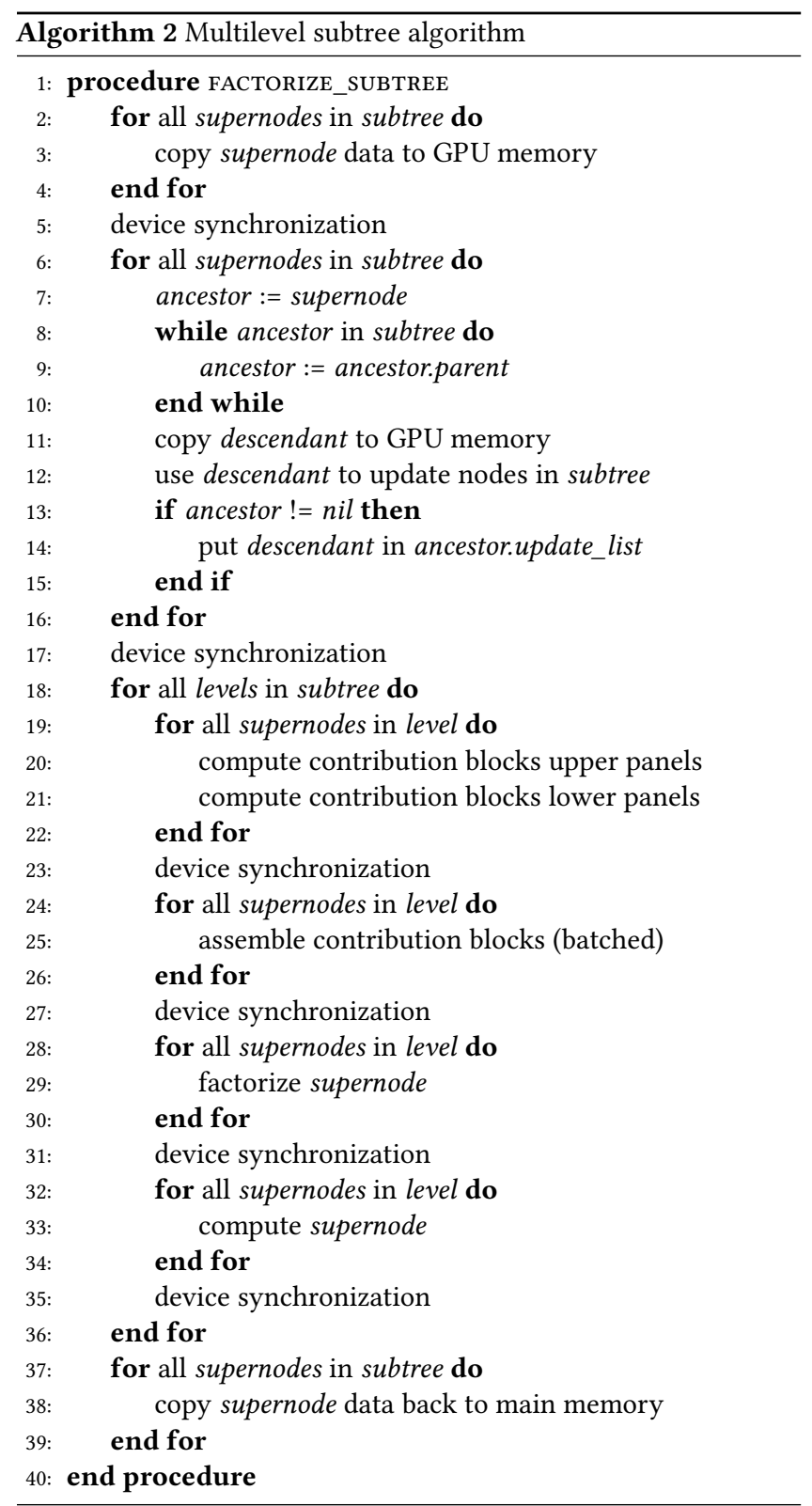

corresponds to a column panel (named "supernode"), which is made of a symmetric matrix and a rectangular matrix. Fig. 1 depicts the construction of a supernode. During the factorization, each supernode goes through the following steps:

- After all descendants are factorized, the supernode is updated by all its relevant descendants.

- The upper symmetric matrix is factorized with a dense Cholesky factorization, and yields a lower triangular matrix.

- The lower rectangular part of the supernode is solved with a triangular solve.

The update operation of supernodal Cholesky algorithm [6] is shown in Fig. 2. The solid circles in the figure represent the updating of the target supernode $s$ by its descendant supernode $d$. Though 


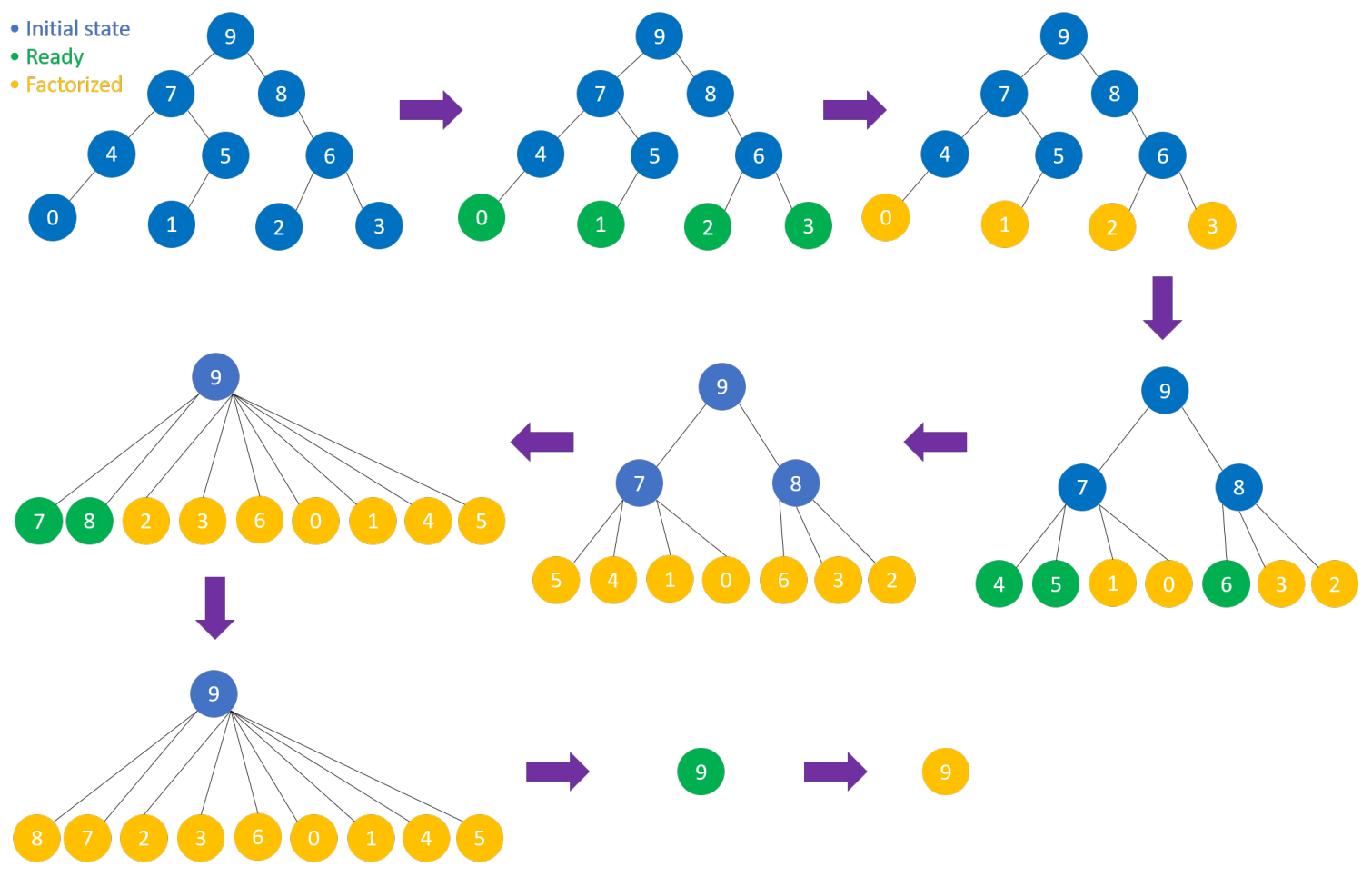

Figure 3: Workflow of left-looking supernodal Cholesky

grey circles are logically nonzero, these do not represent updating of $s$.

In CHOLMOD's implementation, the descendants do not update their ancestor individually. Instead, a contribution block is computed using the descendants, and then added to the ancestor supernode (we will call this operation "assemble").

The left-looking Cholesky requires a supernode to be updated by all its relevant descendants before it is factorized. If two supernodes have no inter dependency, it is possible to process them independently and in parallel. CHOLMOD [2] baseline algorithm (v4.5.3) (and other single-threaded algorithms) first sorts the nodes in ascending order with respect to the column index and then factorizes them. This strategy prevents violations of data dependencies since no lower-indexed columns depend on higher-indexed columns. Hence no scheduler is required in this case as the workflow is simple.

Fig. 3 depicts the workflow of the left-looking supernodal Cholesky algorithm. Here, a node stores a list of its descendant nodes that are required for updating it. Initially the list is empty. After the factorization of a supernode, the supernode is added to the list of descendant nodes of its parent. After a factorized supernode $p$ updates its ancestor $q$, CHOLMOD finds the parent of $q$ : $r$, removes $p$ from $q$ 's list and adds it to $r$ 's list.

The baseline algorithm is efficiently implemented on a hybrid processor comprising CPUs and GPUs. The bottleneck in this implementation is data transfers happening over the PCI Express bus. Such data is primarily transferred between main memory and the memory of the GPU to update an ancestor. Hence using the GPU benefits only those kernels involving sufficiently large computations, preventing the GPU from accelerating some portion of the factorization.

\section{SUBTREE ALGORITHM}

Rennich, Stosic and Davis [14] developed the subtree algorithm deriving it from the baseline algorithm. The subtree algorithm avoids the data transfers of the baseline algorithm during ancestor updates. This is done firstly, by copying a branch of the elimination tree into GPU memory, secondly by factorizing the supernodes in the branch in batches entirely on the GPU, and finally, copying the factorized subtree back to the main memory. Thus, unlike the baseline algorithm, the subtree algorithm does not transfer supernodes back and forth between the GPU memory and the main memory. This improves performance as communication over PCIe is reduced. Further, the subtree algorithm launches BLAS calls such as DSYRK and DGEMM in batches. This improves performance by reducing the launch latencies of these function calls, as well as by increasing the concurrency of DGEMM kernels.

The subtree algorithm first forms groups of supernodes and stores these groups in an array of "levels" (this is not the same "level" as in "multilevel" subtree algorithm). The supernodes stored at a particular level are independent and depend only on supernodes stored in previous levels. Thus, no dependencies are violated when factorization happens a level at a time, from lower to higher levels.

The subtrees are created and then actual factorization takes place. We define the maximum size of a subtree as the capacity of GPU memory. Our algorithm accepts multiple subtrees or "subforests" as valid input. The subtrees are created such that each subtree 


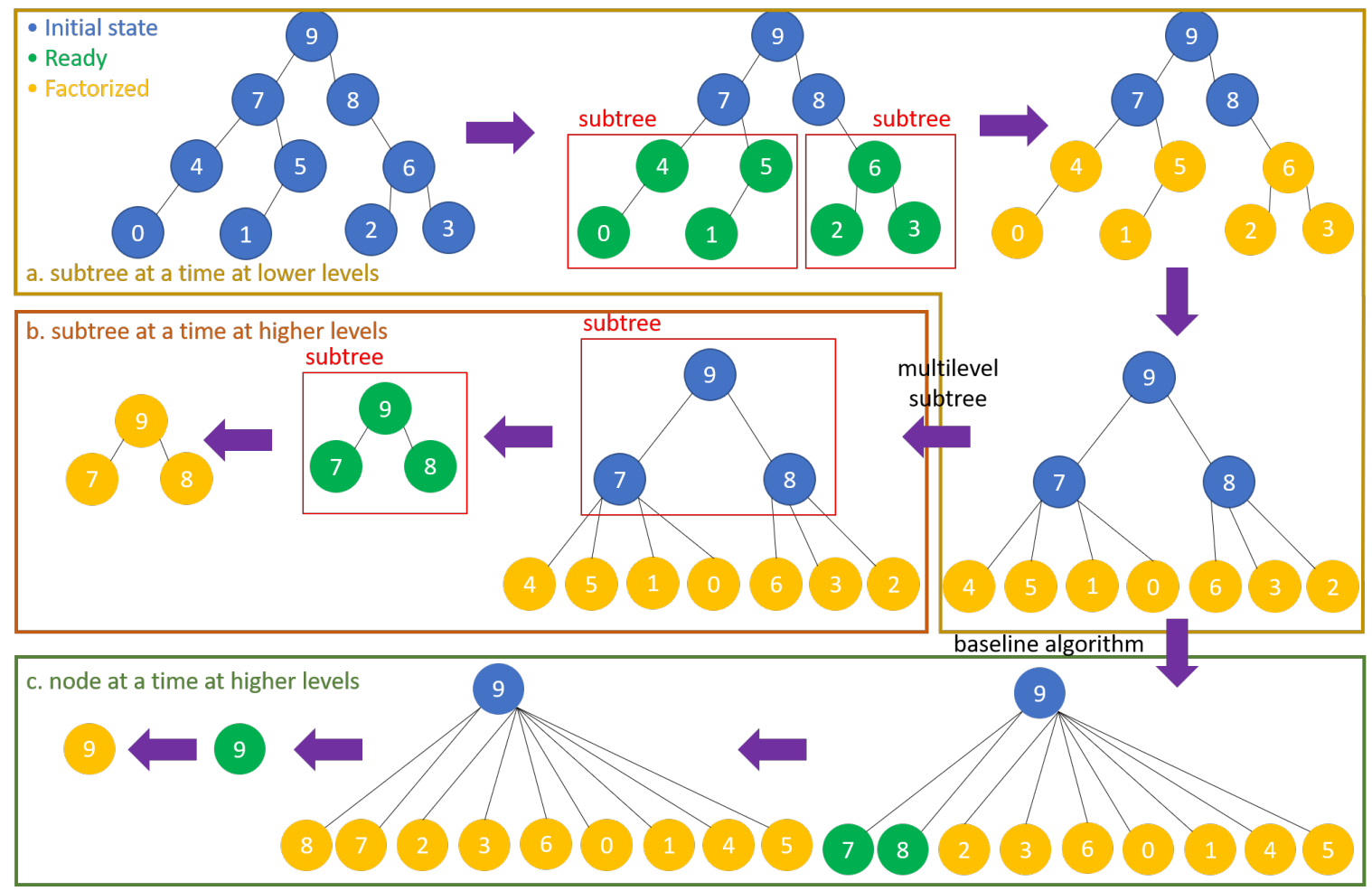

Figure 4: Workflow of single level subtree algorithm $(a+c)$ and multilevel subtree algorithm $(a+b)$

is a subforest of the original elimination tree. The supersets of these subtrees are, in fact, also sub-forests of the elimination tree. However the supersets exceed the maximum allowable size.

After the construction of subtrees, these subtrees are factorized as follows. Firstly, the supernodes in the subtree are copied into the GPU memory. Secondly, each supernode is factorized with the factorization happening level by level, starting from the supernodes at the lowest level. The computation for factorization involves batched calls to the BLAS routines DSYRK, DGEMM, DPOTRF and DTRSM. Finally, when the factorization of the last level of supernodes completes, the results are copied back from GPU memory to the main memory.

Algorithm 1 illustrates the implementation of the subtree factorization.

In case of large matrices, it is possible that the entire matrix cannot be stored in the GPU memory. In that case, the subtrees will be processed as usual but there will remain supernodes at higher levels that cannot be factorized. These supernodes form a tree that we call a "root tree". Our algorithm will use the baseline algorithm, to factorize the root tree.

Fig. 4 depicts the workflow of the subtree algorithm. Note that after factorizing the subtree, the program may choose between the baseline algorithm and the multilevel subtree algorithm (which we will discuss later).

\section{MULTILEVEL SUBTREE ALGORITHM}

The introduction of the subtree algorithm brings great improvement to the performance, however, in the initial subtree-enabled
CHOLMOD implementation, the subtree algorithm is only applied to the lower levels of the elimination tree.

Our multilevel subtree algorithm tries to create even more subtrees, by applying the same approach for the entire tree. This will take the form of a while loop which repeats until either the matrix is fully factorized or no more subtrees can be constructed.

All "non-leaf" subtrees are processed by the algorithm in the second loop. These subtrees will have their descendants, and thus will have data dependency on other subtrees, i.e. they need to be updated by their descendant subtrees before being factorized.

Supernodes on higher levels of the elimination tree tend to have a large number of descendants. Our experiments have shown that usually after the first loop of the subtree algorithm, each remaining unfactorized supernode will have a set of descendants whose total size exceeds the capacity of our GPU memory. Most of these descendants are supernodes that are already factorized in previous subtree factorizations, but have not yet updated all of their respective ancestor supernodes. To address this issue, we exclude supernodes that are already factorized in previous loops from newly constructed subtrees. Those supernodes will be stored in the main memory, and copied to the GPU memory only when they are needed in an update operation.

To hide the host $\leftrightarrow$ device communications incurred during the inter-subtree updates, two blocks of memory are allocated on the GPU, so that while the descendant supernode stored in one block of memory is used in the updates, the next descendant can be copied into the other at the same time. 

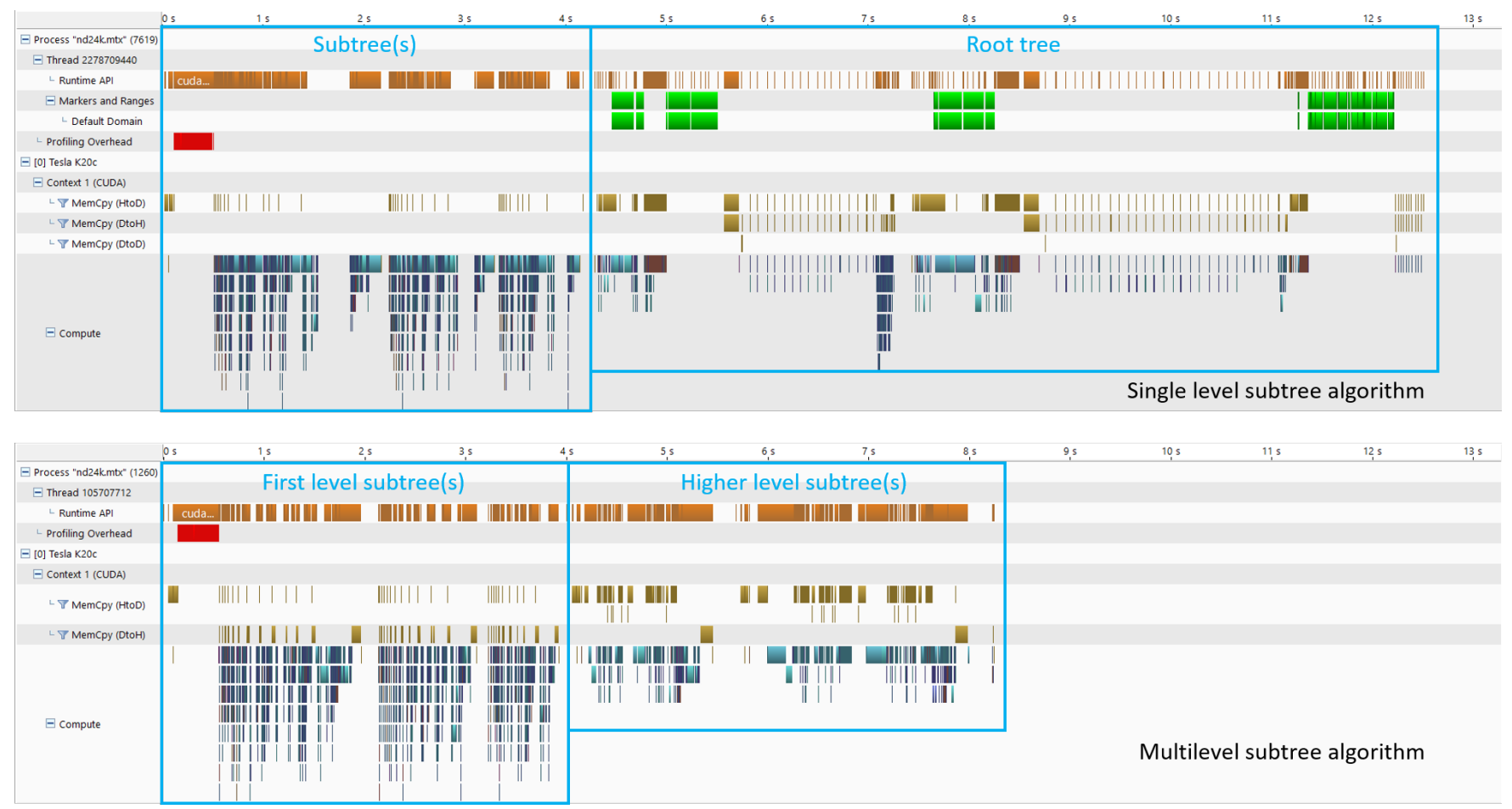

Figure 5: Timing graphs for factorization of matrix nd24k (from SuiteSparse collection) on a single K20c GPU using single level subtree algorithm versus multilevel subtree algorithm. Performance for factorizing the first level subtrees increased due to pipelining. Performance for factorizing higher level nodes (in the form of either the root tree or subtrees) increased due to application of multilevel subtree algorithm.

Fig. 4 also depicts the workflow of the second level of our multilevel subtree algorithm. First of all, subtrees are created from the unfactorized supernodes.

The first step of the factorization of a "non-leaf" subtree is the update operations. Before their factorization, supernodes in the subtree each already have some of their descendants in the update list. We go through those update lists, and use descendant nodes in them to update all supernodes in the subtree. The subtree algorithm speeds up these updates by reducing the number of host-to-device copies because the baseline algorithm would require the descendant to be copied to the GPU memory each time it updates an ancestor (different ancestor supernodes are not present in the GPU simultaneously, and the descendant needs to be overwritten to make room for other descendants of the current supernode), while in the subtree algorithm, the descendant can update all supernodes in the subtree after being copied to the GPU memory. After each descendant updates the subtree, the descendant is detached from the current update list it is in, and put into the update list of its ancestor node, who is also the parent of the subtree's root (or one of the roots if the "subtree" is a subforest).

After the updates the "non-leaf" subtree will become a "leaf", and can be factorized the same way as first-level subtree.

Except requiring update operations before factorizing subtrees, the multilevel subtree algorithm is very similar to the single level subtree algorithm. Algorithm 2 illustrates the implementation of the multilevel subtree factorization on higher levels of the elimination tree.
One may see that the multilevel subtree algorithm is, in a sense, similar to the baseline algorithm, except that the subtree algorithm has much larger "supernodes" (subtrees). The subtree algorithm is also less restrictive on the "supernodes" because in the baseline algorithm supernodes are required to be dense matrices, but the subtree algorithm exploits the sparsity of the matrix and stores a sparse "supernode" in the GPU memory.

Fig. 5 is the timing graphs for the single level subtree algorithm and the multilevel subtree algorithm respectively. The first loop of the multilevel subtree algorithm is essentially identical to the subtree part of the single level subtree algorithm, but since in the multilevel subtree algorithm we implemented an additional pipeline which allows more overlapping of computations and data transfers, the resulting performance is slightly better than the subtree part of the single level subtree algorithm. The baseline algorithm consists of numerous small PCIe transfers and small CUDA kernel calls scattering sparsely over the entire factorization of the root tree. The idle time between these operations becomes a significant performance drawback. The multilevel subtree algorithm partially overcomes this drawback by utilizing batching, which results in a tighter distribution of communications and numerical computations. Note that the CPU does not take part in the factorization of subtrees, but the performance gained through batching still outweighs the loss of the CPU's computing power. 
Table 1: Test matrices used for comparing (Single GPU method) and MAGMA

\begin{tabular}{|l|l|r|r|}
\hline matrix & problem type & dimension & nonzeros \\
\hline Emilia_923 & structural & 923,136 & $40,373,538$ \\
Fault_639 & structural & 638,802 & $27,245,944$ \\
Flan_1565 & structural & $1,564,794$ & $114,165,372$ \\
Geo_1438 & structural & $1,437,960$ & $60,236,322$ \\
Hook_1498 & structural & $1,498,023$ & $59,374,451$ \\
Serena & structural & $1,391,349$ & $64,131,971$ \\
StocF-1465 & computational & $1,465,137$ & $21,005,389$ \\
& fluid dynamics & & \\
audikw_1 & structural & 943,695 & $77,651,847$ \\
bone010 & model reduction & 986,703 & $47,851,783$ \\
nd24k & 2D/3D problem & 72,000 & $28,715,634$ \\
\hline
\end{tabular}

\section{BATCHED SPARSE CHOLESKY FACTORIZATION}

Till now all the work that has been done is factorizing one sparse matrix at a time. In this section we will look into the factorization of multiple matrices simultaneously.

The Cholesky factorization of multiple matrices $A_{1}, A_{2}, \cdots, A_{n}$ can be viewed as a Cholesky factorization of matrix $A$, where $A$ is a matrix constructed by merging $A_{k}(k=1,2, \cdots, n)$.

$$
\text { Let } A=\left[\begin{array}{llll}
A_{1} & & & \\
& A_{2} & & \\
& & \ddots & \\
& & & A_{n}
\end{array}\right] \text { and assume that } A_{k}=L_{k} L_{k}^{T}
$$

$(k=1,2, \cdots, n)$ where $L_{k}$ is lower triangular. It is clear to see that $A=L L^{T}$ where $L=\left[\begin{array}{llll}L_{1} & & & \\ & L_{2} & & \\ & & \ddots & \\ & & & L_{n}\end{array}\right]$ and $L$ is lower triangular.

Based on this property of the Cholesky factorization, our method to factorize matrices in batches is very straightforward: arrange the subject matrices diagonally to form a larger matrix, and factorize the new matrix instead.

The elimination tree of $A$ will be a forest composed of the elimination trees of $A_{1}, A_{2}, \cdots, A_{n}$. Since $A_{1}, A_{2}, \cdots, A_{n}$ have no dependency on each other, it is possible to factorize them in parallel. More precisely, since the supernodes and subtrees from different matrices have no data dependency, if multiple GPUs (or multiple threads) are present, the batched factorization allows greater flexibility in the scheduling of the workflow than serial non-batched factorizations.

The batched sparse Cholesky factorization also works well with the subtree algorithm. The merging of two sparse matrices can result in subtrees containing supernodes from different matrices, allowing larger subtrees to be constructed.

Fig. 8 shows an example in which the batched factorization algorithm creates larger subtrees using supernodes from different matrices. This will allow the algorithm to more effectively exploit the computing power of GPUs.

Fig. 7 shows the comparison between the factorization of matrix nd24k on two K20c GPUs and the batched factorization of $2 \mathrm{x}$ nd24k on two K20c GPUs. The latter takes less than double the former's time consumption to finish, which is a demonstration that batched sparse Cholesky factorization may allow us to save overall factorization time of multiple matrices.

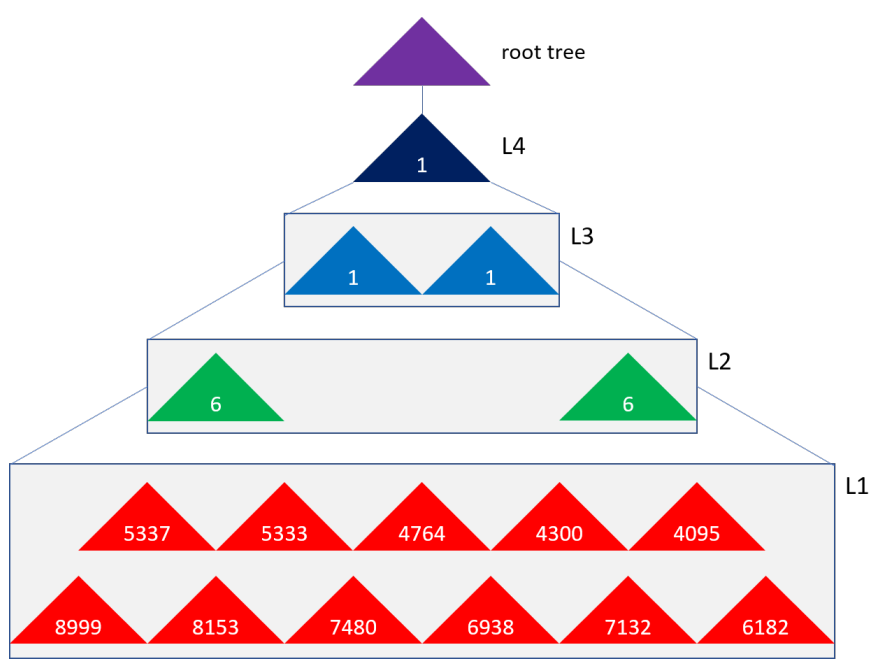

Figure 6: Structure of matrix Geo_1438 (from SuiteSparse collection). No performance gain on $\mathrm{L} 3$ and $\mathrm{L} 4$ subtrees since each subtree contains only one supernode.

\section{EXPERIMENTAL RESULTS}

In this section we present the experiments performed to evaluate the performance of our algorithm. Due to the availability of equipment, our experiments were carried out on different platforms. Table 1 provides summary information about the matrices used in our performance measurements. These matrices were chosen from the SuiteSparse Matrix Collection.

Experiments for the multilevel subtree algorithm were performed on a platform with a dual socket AMD Opteron Processor 6168 CPU and two NVIDIA Tesla K20c GPUs. We compare the performance of our multilevel subtree algorithm with two other versions of CHOLMOD: CHOLMOD 4.6.0-beta, the single level subtree algorithm [14] and the pipeline enhanced version [16] of CHOLMOD 4.6.0-beta. Note that our implementation of the multilevel subtree algorithm also has the pipelines enabled.

Fig. 9 shows the performance when only one GPU is used. It can be seen that the multilevel subtree algorithm achieves performance of up to $2.43 \mathrm{x}$ vs. the single level subtree algorithm, and performance of up to $1.42 \mathrm{x}$ vs. the pipeline enhanced version. For 9 of the 10 matrices, using the multilevel subtree algorithm along with pipeline led to enhancements in performance compared to the single level subtree algorithm. The average speedup is $1.59 \mathrm{x}$ over the single level subtree algorithm.

Note that the multilevel subtree algorithm does not bring a performance boost for all matrices. Geo_1438 is an example in which the application of the multilevel subtree method results in a performance loss. Since the effectiveness of subtrees relies on batching, if there is only one supernode in a subtree, there will be no performance gain, and the extra overhead introduced in the subtree method may slow the entire algorithm down. 

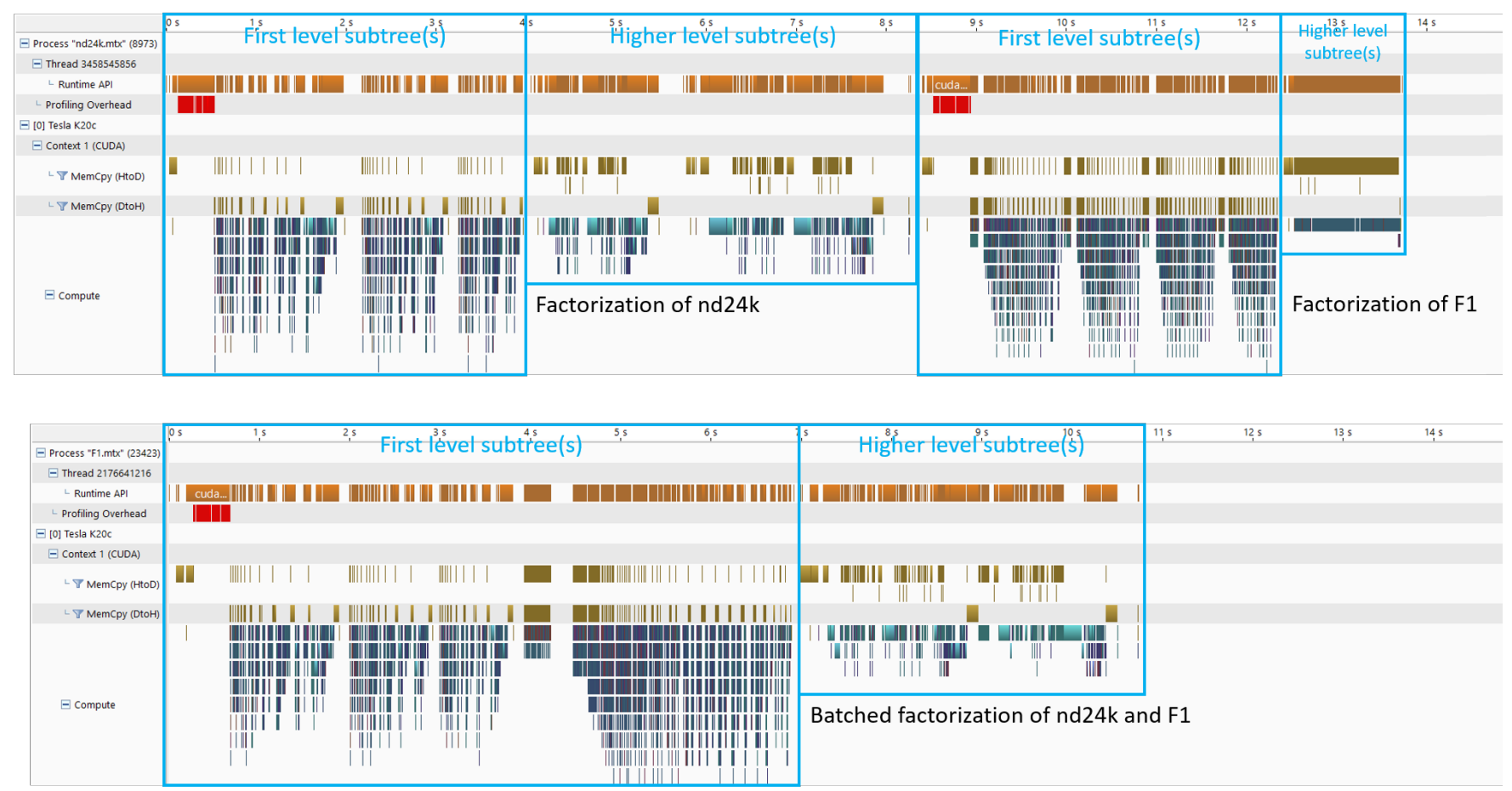

Figure 7: Timing graph comparison between one-by-one matrix factorization and batched factorization. Total factorization time is decreased when using batched factorization.

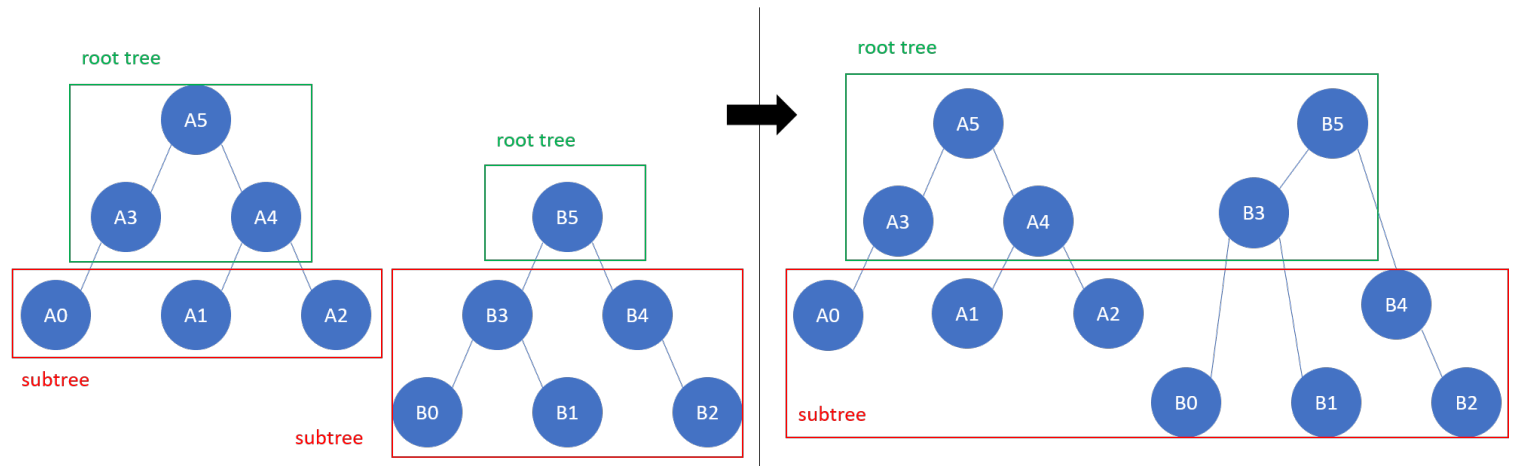

Figure 8: Potential increase in subtree size when using batched factorization

Fig. 6 depicts the structure of the matrix Geo_1438, where each triangle stands for either a subtree or the root tree. The number in each triangle shows the number of supernodes in the subtree. Since the subtrees in the third and the fourth level each contains only one supernode, they are not able to provide any performance boost. In this case the only possible performance gain over the single level subtree algorithm lies in the second level. However the experiment result shows that it is not enough to offset the loss in the third and the fourth level. Using GPUs with larger memory may address this issue.

Fig. 10 adds the data of the performance when both GPUs are used. When using two GPUs the performance of the multilevel subtree algorithm is worse than the single level subtree algorithm. The cause behind this is not yet confirmed.

Experiments for the batched sparse Cholesky factorization were performed on a platform with a dual socket Intel Xeon E5-2695 v2 CPU and eight NVIDIA Tesla K80 GPUs. Our goal is to show that factorizing multiple matrices in batch is faster than factorizing them serially. We first look into the case where the matrices in the batch are identical, then for each matrix $A$, we only need to compare the Gflops of the factorization of a single $A$ with the Gflops of batched factorization of multiple $A$ 's. Performance was measured using both the single level subtree algorithm and the multilevel subtree algorithm. The following experiments cover only the batched factorization of multiple identical matrices. 


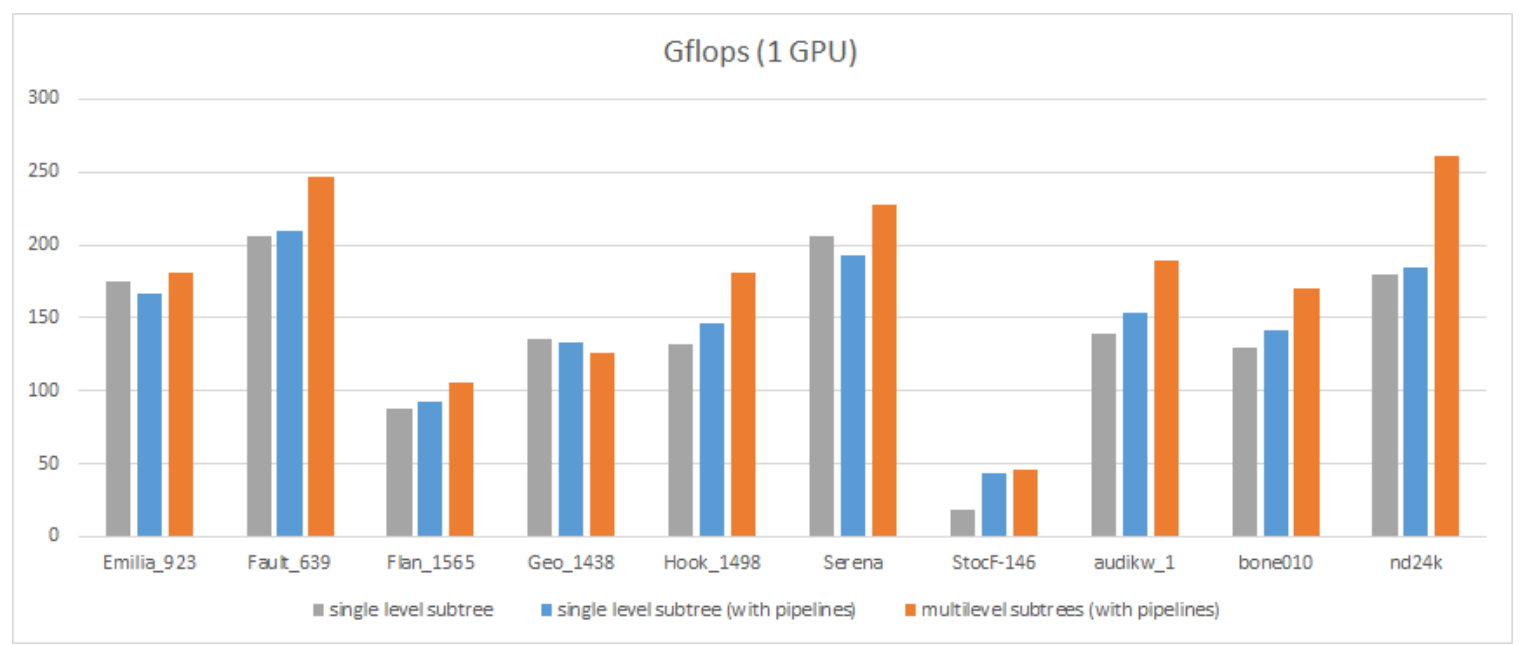

Figure 9: Performance comparison between baseline, single level subtree and multilevel subtree algorithm on single GPU

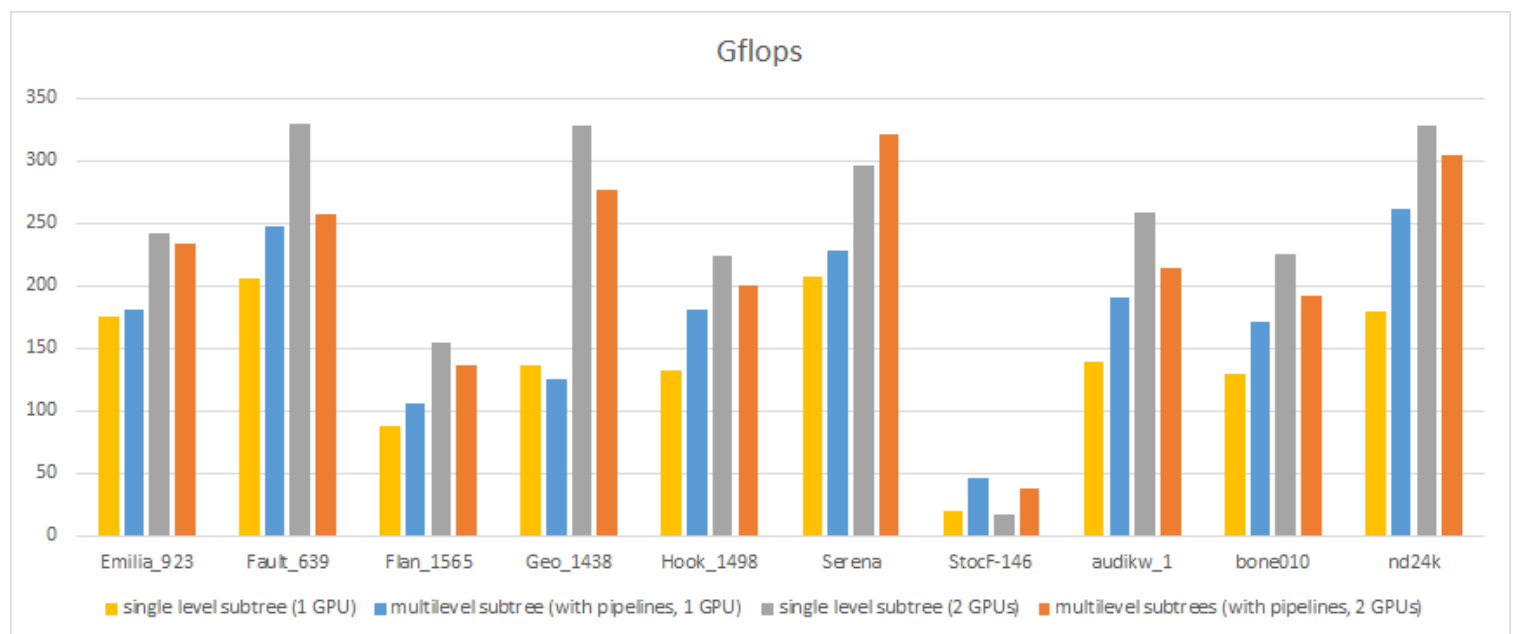

Figure 10: Performance comparison between baseline, single level subtree and multilevel subtree algorithm on single GPU and two GPUs

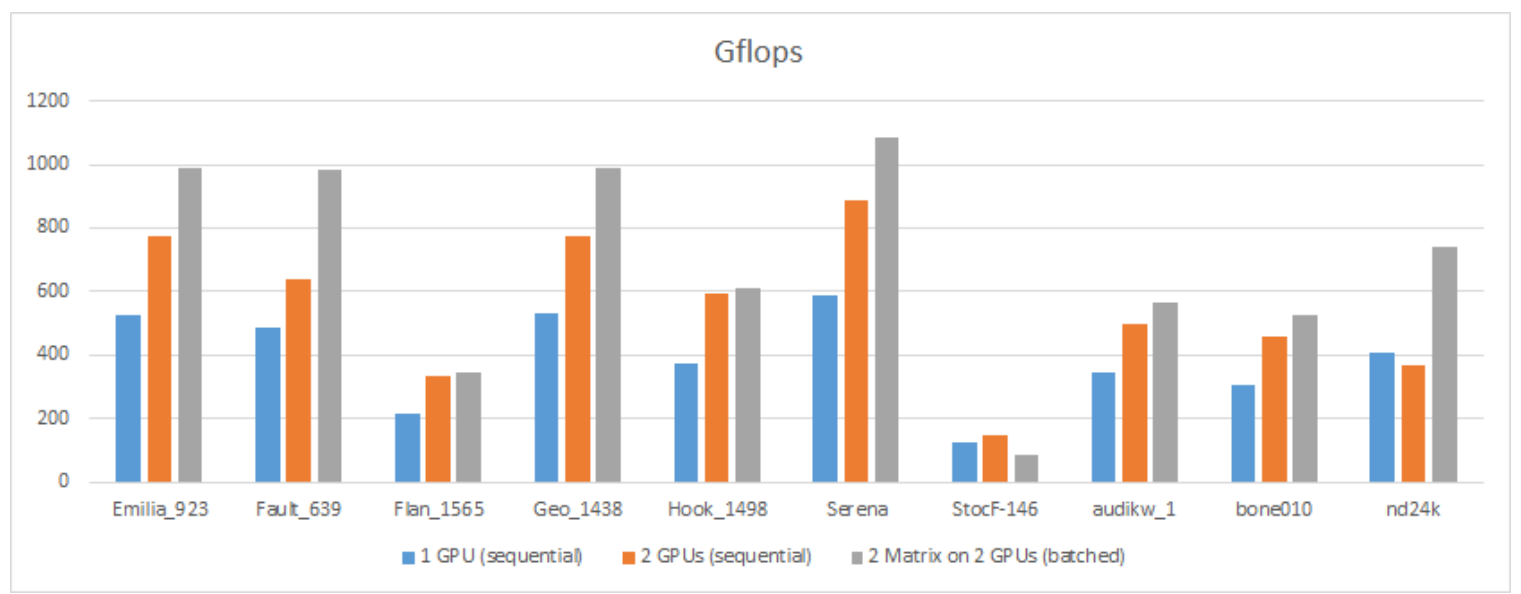

Figure 11: Batched Cholesky factorization performance versus sequential matrices factorization on GPUs (2 GPUs) 


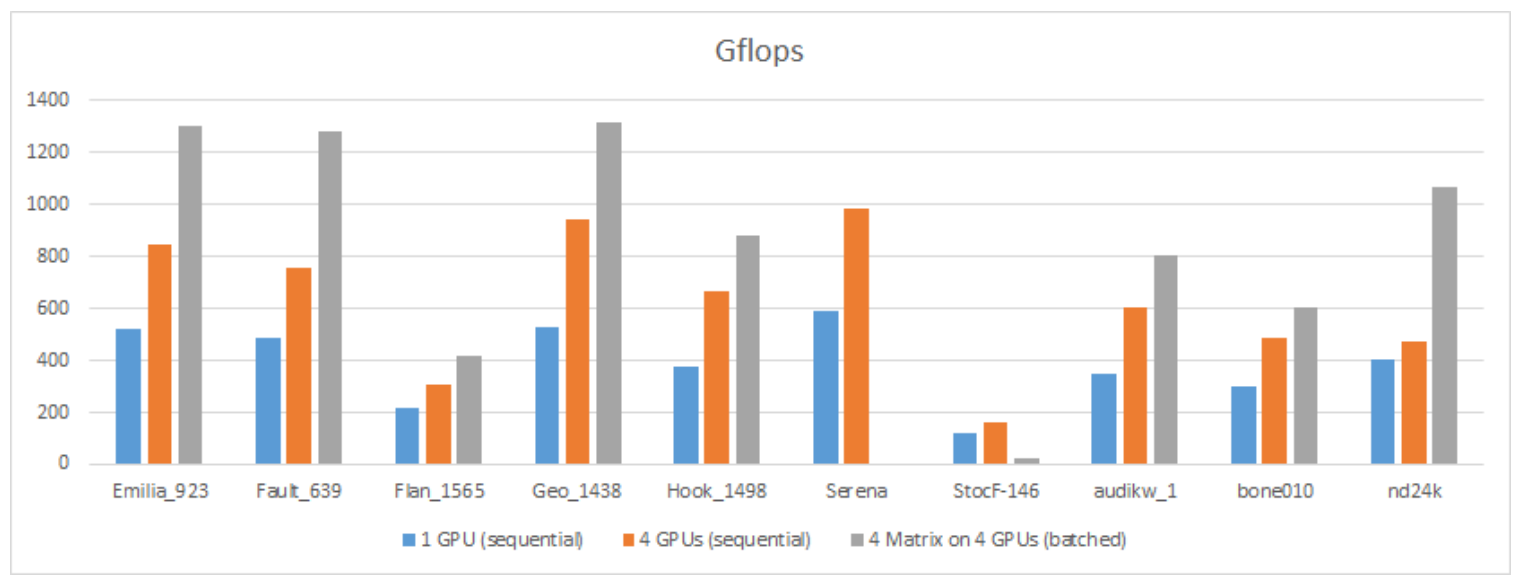

Figure 12: Batched Cholesky factorization performance versus sequential matrices factorization on GPUs (4 GPUs)

Fig. 11 and Fig. 12 show the experiments results for batched sparse Cholesky factorization (multilevel subtree algorithm) (data for batched factorization of $4 \mathrm{x}$ matrix Serena are missing). With 2 GPUs and 2 matrices to factorize, the batched factorization is able to improve the performance by up to $53.9 \%$. With 4 GPUs and 4 matrices to factorize, the batched factorization is able to improve the performance by up to $125.0 \%$.

\section{CONCLUSIONS}

In this paper we present the multilevel subtree algorithm and the batched sparse Cholesky factorization. The multilevel subtree algorithm works by extending the subtree algorithm to create subtrees on higher levels of the elimination tree. The size of "non-leaf" subtrees are reduced by excluding factorized supernodes. Experimental results demonstrate up to $143 \%$ performance improvement over the single level subtree algorithm, or up to $42 \%$ improvement over the pipeline enhanced single level subtree algorithm.

The batched sparse Cholesky factorization works by factorizing a larger sparse matrix which is a diagonal combination of subject matrices in the batch. The batched Cholesky factorization of sparse matrices potentially increases possible parallelism, increases scheduling flexibility, and allows larger subtree sizes. In our experiments the batching brings up to $140 \%$ increase in performance when factorizing two or four identical matrices. It is also observed that the effect of factorizing in batches is generally better when more GPUs are used. When factorizing multiple different matrices in batches the results are not ideal, but we have not yet confirmed if the choice of platform has negatively affected the experiments.

With these techniques (pipelining, subtree algorithm, multilevel subtree algorithm, batch factorization) applied to the sparse Cholesky factorization, we are now working to apply them to sparse QR and sparse LU.

\section{ACKNOWLEDGMENTS}

This material is based upon work supported by the National Science Foundation under Grant No. CNS 1514116. Any opinions, findings, and conclusions or recommendations expressed in this material are those of the author(s) and do not necessarily reflect the views of the National Science Foundation.

\section{REFERENCES}

[1] C. C. Ashcraft, R. G. Grimes, J. G. Lewis, B. W. Peyton, and H. D. Simon. 1987. Progress in Sparse Matrix Methods for Large Linear Systems on Vector Supercomputers. 1, 4 (1987), 10-30.

[2] Yanqing Chen, Timothy A Davis, William W Hager, and Sivasankaran Rajamanickam. 2008. Algorithm 887: CHOLMOD, supernodal sparse Cholesky factorization and update/downdate. ACM Transactions on Mathematical Software (TOMS) 35,3 (2008), 22

[3] G.W. Collins and NASA Astrophysics Data System. 2003. Fundamental Numerical Methods and Data Analysis. George W. Collins, II.

[4] Timothy A Davis. 2006. Direct methods for sparse linear systems. SIAM.

[5] Timothy A Davis and Yifan Hu. 2011. The University of Florida sparse matrix collection. ACM Transactions on Mathematical Software (TOMS) 38, 1 (2011), 1.

[6] Timothy A Davis, Sivasankaran Rajamanickam, and Wissam M Sid-Lakhdar. 2016. A survey of direct methods for sparse linear systems. Acta Numerica 25 (2016), 383-566.

[7] Tingxing Dong, Azzam Haidar, Stanimire Tomov, and Jack Dongarra. 2014. A fast batched Cholesky factorization on a GPU. In Parallel Processing (ICPP), 2014 43rd International Conference on. IEEE, 432-440.

[8] Azzam Haidar, Tingxing Dong, Piotr Luszczek, Stanimire Tomov, and Jack Dongarra. 2015. Batched matrix computations on hardware accelerators based on GPUs. Intl. F. of High Performance Computing Applications 29, 2 (2015), 193-208.

[9] Azzam Haidar, Tingxing Tim Dong, Stanimire Tomov, Piotr Luszczek, and Jack Dongarra. 2015. A framework for batched and GPU-resident factorization algorithms applied to block householder transformations. In International Conference on High Performance Computing. Springer, 31-47.

[10] J. D. Hogg, E. Ovtchinnikov, and J. A. Scott. 2016. A sparse symmetric indefinite direct solver for GPU architectures. 42 (2016), 1:1-1:25. http://doi.acm.org/10. $1145 / 2756548$

[11] Jakub Kurzak, Hartwig Anzt, Mark Gates, and Jack Dongarra. 2016. Implementation and tuning of batched Cholesky factorization and solve for NVIDIA GPUs. IEEE Transactions on Parallel and Distributed Systems 27, 7 (2016), 2036-2048.

[12] X. Lacoste, P. Ramet, M. Faverge, Y. Ichitaro, and J. Dongarra. 2012. Sparse direct solvers with accelerators over DAG runtimes. Technical Report RR-7972. INRIA, Bordeaux, France. 11 pages. http://hal.inria.fr/hal-00700066

[13] Steven C Rennich, Darko Stosic, and Timothy A Davis. 2014. Accelerating sparse Cholesky factorization on GPUs. In Proceedings of the Fourth Workshop on Irregular Applications: Architectures and Algorithms. IEEE Press, 9-16.

[14] Steven C Rennich, Darko Stosic, and Timothy A Davis. 2016. Accelerating sparse Cholesky factorization on GPUs. Parallel Comput. 59 (2016), 140-150.

[15] Meng Tang, Mohamed Gadou, and Sanjay Ranka. 2017. A Multithreaded Algorithm for Sparse Cholesky Factorization on Hybrid Multicore Architectures. Procedia Computer Science 108 (2017), 616-625.

[16] Meng Tang, Mohamed Gadou, Steven Rennich, Timothy A Davis, and Sanjay Ranka. 2018. Optimized Sparse Cholesky Factorization on Hybrid Multicore Architectures. Fournal of Computational Science (2018).

[17] Oreste Villa, Massimiliano Fatica, Nitin Gawande, and Antonino Tumeo. 2013. Power/performance trade-offs of small batched LU based solvers on GPUs. In European Conference on Parallel Processing. Springer, 813-825.

[18] Sencer Nuri Yeralan, Timothy A Davis, Wissam M Sid-Lakhdar, and Sanjay Ranka. 2017. Algorithm 980: Sparse QR Factorization on the GPU. ACM Transactions on Mathematical Software (TOMS) 44, 2 (2017), 17. 